Case Report

\title{
Hepatotoxicity due to Clindamycin in Combination with Acetaminophen in a 62-Year-Old African American Female: A Case Report and Review of the Literature
}

\author{
Jerome Okudo ${ }^{1}$ and Nwabundo Anusim ${ }^{2}$ \\ ${ }^{1}$ School of Public Health, University of Texas, 1200 Pressler Street, Houston, TX 77030, USA \\ ${ }^{2}$ Department of Medicine, Saint Joseph Regional Medical Center, 5215 Holy Cross Parkway, Mishawaka, IN 46545, USA
}

Correspondence should be addressed to Jerome Okudo; jeromeokudo@yahoo.com

Received 15 January 2016; Revised 16 May 2016; Accepted 6 June 2016

Academic Editor: Fumio Imazeki

Copyright (C) 2016 J. Okudo and N. Anusim. This is an open access article distributed under the Creative Commons Attribution License, which permits unrestricted use, distribution, and reproduction in any medium, provided the original work is properly cited.

\begin{abstract}
Clindamycin is a bacteriostatic lincosamide antibiotic with a broad spectrum. Side effects include nausea, vomiting, diarrhea, and metallic taste; however, hepatotoxicity is rare. The incidence is unknown. It is characterized by increases in aspartate and alanine transaminases. There may be no symptoms and the treatment is to stop the administration of clindamycin. We have described a 62-year-old African American female medicated with acetaminophen and clindamycin who had initially presented to the dental clinic for the evaluation of gum pain following tooth extraction. She had significantly increased levels of liver transaminases, which trended downwards on quitting the medication.
\end{abstract}

\section{Introduction}

Clindamycin is a common bacteriostatic antibiotic with wide coverage against several organisms [1-3]. It is useful in the treatment of oral infections and is quickly distributed in the body following oral administration. It is metabolized and excreted by the liver.

While clindamycin has many side effects, hepatotoxicity is a rare culprit in liver damage $[4,5]$. There is limited medical literature supporting the role of clindamycin in liver damage even though apoptosis plays a role. Damage from clindamycin causes significant increases in both aspartate transaminase and alanine transaminase and liver biopsy may demonstrate damage to the portal system, which may show reversal to baseline when clindamycin administration is stopped $[4,5]$. We have discussed a patient who received oral clindamycin for a dental infection and subsequently developed clindamycin hepatotoxicity.

\section{Case Report}

A 62-year-old African American female with a history of tobacco use, alcohol use, hypertension, and hyperlipidemia presented to the hospital with severe pains in her gum nine days following a tooth extraction at sites 19 and 30 . She self-medicated with several alternated tablets of $750 \mathrm{mg}$ acetaminophen every six hours and ibuprofen $400 \mathrm{mg}$ prn but her gum pain was unrelenting. In the interim, she saw her primary care physician who prescribed $450 \mathrm{mg} 6$ hourly clindamycin for ten days for a possible dental infection. In spite of her compliance to clindamycin, after five days of treatment, her gum pain persisted and was severe. It became evident that she would require hospitalization. At presentation at the emergency department, physical examination was unremarkable; the maxillofacial surgeon made an assessment of alveolar osteitis and chlorhexidine mouthwash was started for the patient. Upon initial evaluation of the laboratory results in the emergency department, it was found that the patient had the following results: alanine aminotransferase (ALT) was $423 \mathrm{IU} / \mathrm{L}$ (normal range 0-40); aspartate aminotransferase (AST) was 338 IU/L (normal range 5-40); her total bilirubin and INR levels were within reference ranges, gamma-glutamyl transpeptidase (GGT) was $179 \mathrm{IU} / \mathrm{L}$ (normal range of 10-64); and alkaline phosphatase (ALP) was $321 \mathrm{IU} / \mathrm{L}$ (normal range 40-100). Her acetaminophen level was $31 \mathrm{mcg} / \mathrm{mL}$ and alcohol level was $7 \mathrm{mg} / \mathrm{dL}$. At this time, there was suspicion of acetaminophen poisoning 
and poison control was notified and she was started on acetylcysteine 21-hour dose regimen; this was extended after 48 hours per protocol. On the 7th day on oral clindamycin, there was suspicion of clindamycin toxicity as indicated by her latest ALT (1579) and AST (1512) results which had trended upwards; additional history was sought from the patient. There was no fever, chills, fatigue, anorexia, weakness, nausea, abdominal pain, dark-colored urine, jaundice, pruritus, lymphadenopathy, or rash. There was neither history of chronic liver disease nor hypersensitivity features in the patient. There were also no hypersensitivity features in the patient. Her other blood tests included glucose, cholesterol, triglycerides, protein, albumin, uric acid, blood urea nitrogen (BUN), and serum creatinine and they were determined to be within normal ranges. Hepatitis A antibody IgM, B core IgM, surface antigen, hepatitis C, HIV, cytomegalovirus, Epstein Barr virus, and antinuclear antibody screen (ANA) were negative. We did not test hepatitis $\mathrm{E}$ virus in this patient; however, the patient had not recently traveled to any regions in Africa, Asia, or Central America where the virus is well known. A review of the patient by the gastroenterology team was performed and a Doppler of the hepatic vein, hepatitis panel (already performed), and a liver ultrasound scan were ordered to rule out Budd-Chiari syndrome, acute hepatitis, and biliary obstruction; however, all tests were negative. A liver biopsy was not performed for financial reasons. During this time, ALT and AST values were monitored continuously with very high values. By day 9 on clindamycin, the highest values of ALT (1927) and AST (1812) were gotten in the patient and it was suggested that clindamycin be discontinued, and clindamycin was therefore discontinued on day 10 . By the third week, the values of ALT and AST began to trend downwards; ALT (976) and AST (878) were the latest results. During the 4th week, her values had improved to ALT (822) and AST (676). She is being followed up in the clinic on several clinic visits as outpatient until her results return to normal.

\section{Discussion}

Drug-induced liver injury (DILI) is very common and has been reported as being responsible for drug withdrawal from the market. It has been implicated in liver failure. It is imperative to make a clear distinction between DILI caused by overdose and that caused by administration of drug, that is, idiosyncratic DILI. It is also imperative to consider the effect of two main drug categories, one that can lead to severe injury and another that has a low likelihood of causing severe liver damage. To ensure a proper classification of DILI, DILI criteria have long been made available in the medical literature. DILI criteria and classification (adapted from Aithal et al. 2011) will be used in this patient [9-12]. The medical literature has suggested that there should be minimal elements for reporting drug-induced liver injury. Though this is a long list, our case report met more than $90 \%$ of the criteria and has discussed these elements in the case reports. They include the demographics such as sex and age of the patient. Other criteria are drugs and their respective doses, indication, and disease for which the medication was used and other comorbid or concurrent conditions or pertinent past medical history in the patient such as previous drug reactions and liver disease, history of alcohol use, time of onset of event, symptoms, pertinent liver symptoms and signs, detailed medication history prior to the current culprit medication, abnormal laboratory tests and dates reported and baseline liver tests, exclusion of hepatitis by a panel, other liver tests with their course, imaging studies, liver histology, and rechallenge [9-12]. Of all these, the last two were not performed in this patient for financial reasons.

In meeting the DILI criteria, seven major criteria were considered and they include the following: (i) clinical chemistry criteria for drug-induced liver injury (DILI) (two criteria were met even though one criterion is required out of three criteria; they include more than or equal to fivefold elevation above the upper limit of normal for alanine aminotransferase (ALT) and more than or equal to threefold elevation in ALT concentration and simultaneous elevation of bilirubin concentration exceeding $2 \mathrm{x}$ ULN). (ii) clinical pattern of drug-induced liver injury (DILI) which was hepatocellular based on the formula (it is important to bear in mind that $R$ ratios of $>5$ have a high likelihood to be hepatocellular, $<2$ a cholestatic pattern, and between 2 and 5 a mixed pattern of enzymes. If the ALT value is determined to be greater than twice the upper limit of the normal range (ULN) and the alkaline phosphatase is normal, the pattern should be considered hepatocellular and $R$ ratio need not be calculated. Also, if the alkaline phosphatase value is more than twice ULN but the ALT is normal, the pattern should be considered cholestatic, and $R$ ratio may not be calculated. In the RUCAM system, cases determined to be mixed are given scores as if they were cholestatic [9-12]), (iii) DILI severity index which we inferred to be moderate because of the elevated ALT/ALP concentration reaching criteria for DILI and bilirubin concentration $\geq 2 \mathrm{x}$ ULN, (iv) DILI causality assessment which was computed for clindamycin and acetaminophen, respectively, (scores of 10 and 9 were computed, respectively, using the Roussel Uclaf Causality Assessment Method (RUCAM) scale), (v) persistent and chronic drug-induced liver injury (DILI) based on a timeframe beyond three months' follow-up for which the liver transaminases had returned to normal, (vi) drug-associated chronic liver disease (this patient did not display any evidence of chronic liver disease, and for financial reasons a liver biopsy was not performed), and (vii) drug-induced autoimmune hepatitis (ALH). To determine drug induced autoimmune hepatitis, a scoring system using a simplified set of diagnostic criteria will prove useful. A score of $>6$ is obtainable if a liver biopsy is performed for histological evaluation of the liver. In addition, assessment of multiple autoantibodies (antinuclear antibody, smooth muscle cell antibody, liver-kidney microsomal antibodies, and soluble liver/liver-pancreas antibodies), quantitative serum-globulins, and exclusion of viral hepatitis are also required. However, histological evaluation of the liver was not performed due to financial difficulties of the patient.

Many drugs have been known to cause liver toxicity and clindamycin is a rare one [7,13-15]. Very few cases of liver toxicity caused by clindamycin administration have been reported (Table 1). The pharmacokinetics of clindamycin is 


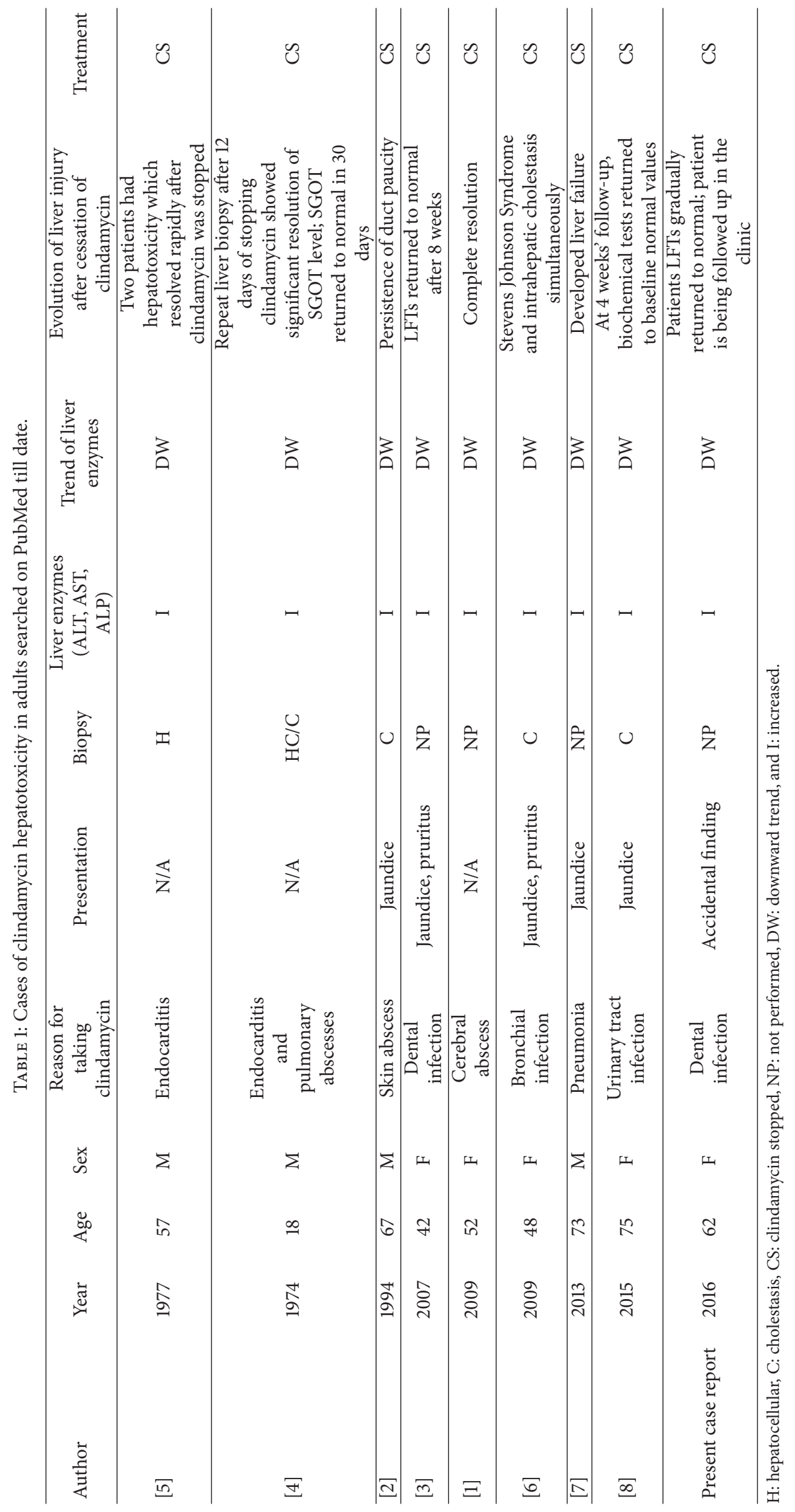


well studied. Oral administration of clindamycin is absorbed almost completely and rapidly so that peak plasma concentration is attained within 45 minutes after it has been orally administered. Absorption is not affected by food. It is well prescribed and oral administration has a high bioavailability. It is also widely distributed in body fluids and tissues. While it is known to diffuse across the placenta, it is not known to diffuse across a healthy blood brain barrier. Majority of clindamycin in the circulation is bound to plasma proteins. It is distributed highly intracellular due to the lipophilic properties. The intracellular concentrations are higher than the extracellular concentrations. It is metabolized in the liver to active metabolites, which include $\mathrm{N}$-dimethyl and sulphoxide, and inactive metabolites and about $4 \%$ in the feces. The half-life is about two and a half hours in children and three hours in adults. It is excreted as biologically active and biologically inactive metabolites in feces, urine, and bile. $10 \%$ of the drug is excreted in the urine as active drug and $4 \%$ in the feces and the rest is excreted as inactive metabolites. It is important to consider special populations such as the elderly and patients with renal and hepatic disease. The half-life, volume of distribution, clearance, and absorption of the drug are not altered by increased age. However, for patients with renal dysfunction, elimination half-life is prolonged. If renal impairment is mild to moderate, there is no reason to reduce the dosage of the medication. In patients who have moderate to severe hepatic impairment, the half-life is also prolonged [16]. Clindamycin is a lincosamide antibiotic known to inhibit bacterial protein synthesis by binding to the $50 \mathrm{~S}$ subunit of the ribosome. It is known to be active against Gram-positive aerobes and anaerobes, Gram-negative anaerobes.

Even though hepatotoxicity is not a well-known side effect, it causes hepatotoxicity in two forms: transient serum aminotransferase elevations usually occurring after several days of high intravenous doses and an acute idiosyncratic liver injury that starts within 1 to 3 weeks of therapy and is usually mild and self-limited $[5,7,8,15]$. Several case reports and reviews have been reported on drug related liver injury for which 176 cases were identified and 39 were due to antimicrobial agents but none was related to clindamycin explaining how rarely studied this entity is $[6,13-15,17]$.

An ultrasound of the liver is important to rule out biliary obstruction [1-3]. This patient self-medicated with acetaminophen for a long period of time and also received clindamycin and both medications are implicated in increased levels of transaminases. To differentiate the liver damage by acetaminophen and clindamycin, clindamycin would cause an increase in both alkaline phosphatase and alanine transaminase described as mixed; however, acetaminophen would cause an increase in alanine transaminase alone [5, 7]. In addition, this patient received $\mathrm{n}$ acetyl cysteine for acetaminophen poisoning but her liver enzymes continued to rise so acetaminophen was not the culprit. While a liver biopsy is important in evaluation, it was not performed in this patient for financial reasons. The following are the likely findings on liver biopsy: significant cholestasis, inflamed portal system, bile duct injury ductopenia, centrilobular, and portal cholestatic hepatitis, without fibrosis or necrosis [3]. Treatment of this condition is to stop clindamycin immediately [1-6]. Symptom resolution, transaminases returning back to baseline on quitting clindamycin, timeline between clindamycin administration and elevation of transaminases, and no other demonstrable causes for rise in liver enzymes are the unique features of this case.

\section{Conclusion}

We reported a case of clindamycin-induced hepatotoxicity, which was an incidental finding. A significant elevation in liver enzymes, which improves when clindamycin is stopped, may aid in the diagnosis of clindamycin-induced hepatotoxicity. The type of injury in this patient was hepatocellular; however, this may vary from one person to another. It is important to look out for patients who take clindamycin because of the potential of more severe presentation of hepatotoxicity. It is important to carefully evaluate patients to make an accurate diagnosis. When making clinical decisions, anchoring is likely. The importance of precise decision making cannot be overemphasized [18].

\section{Competing Interests}

The authors declare that there is no conflict of interests in publishing this paper.

\section{References}

[1] S. Senanayake, "Possible acute hepatotoxicity from oral clindamycin," Australian Prescriber, vol. 32, article 140, 2009.

[2] I. Altraif, L. Lilly, I. R. Wanless, and J. Heathcote, "Cholestatic liver disease with ductopenia (vanishing bile duct syndrome) after administration of clindamycin and trimethoprimsulfamethoxazole," The American Journal of Gastroenterology, vol. 89, no. 8, pp. 1230-1234, 1994.

[3] C. Aygün, O. Kocaman, Y. Gürbüz, Ö. Şentürk, and S. Hülagü, "Clindamycin-induced acute cholestatic hepatitis," World Journal of Gastroenterology, vol. 13, no. 40, pp. 5408-5410, 2007.

[4] M. Elmore, J. P. Rissing, L. Rink, and G. F. Brooks, "Clindamycin-associated hepatotoxicity," The American Journal of Medicine, vol. 57, no. 4, pp. 627-630, 1974.

[5] D. R. Hinthorn, L. H. Baker, D. A. Romig, D. W. Voth, and C. Liu, "Endocarditis treated with clindamycin: relapse and liver dysfunction," Southern Medical Journal, vol. 70, no. 7, pp. 823826, 1977.

[6] J. E. Sahagún Flores, J. A. Soto Ortiz, C. E. Tovar Méndez, E. C. Cárdenas Ochoa, and G. Hernández Flores, "Stevens-Johnson syndrome with intrahepatic cholestasis induced by clindamycin or chlorpheniramine," Dermatology Online Journal, vol. 15, no. 5, article 12, 2009.

[7] M. Z. Bawany, B. Bhutto, W. I. Youssef, A. Nawras, and T. Sodeman, "Acute liver failure: an uncommon complication of commonly used medication," American Journal of Therapeutics, vol. 20, no. 5, pp. 566-568, 2013.

[8] H. Moole, Z. Ahmed, N. Saxena, S. R. Puli, and S. Dhillon, "Oral clindamycin causing acute cholestatic hepatitis without ductopenia: a brief review of idiosyncratic drug-induced liver injury and a case report," Journal of Community Hospital Internal Medicine Perspective, vol. 5, article 28746, pp. 1-5, 2015. 
[9] G. P. Aithal, P. B. Watkins, R. J. Andrade et al., "Case definition and phenotype standardization in drug-induced liver injury," Clinical Pharmacology and Therapeutics, vol. 89, no. 6, pp. 806815, 2011.

[10] E. S. Björnsson and J. H. Hoofnagle, "Categorization of drugs implicated in causing liver injury: critical assessment based on published case reports," Hepatology, vol. 63, no. 2, pp. 590-603, 2016.

[11] V. K. Agarwal, J. G. McHutchison, J. H. Hoofnagle, and DrugInduced Liver Injury Network (DILIN), "Important elements for the diagnosis of drug-induced liver injury," Clinical Gastroenterology and Hepatology, vol. 8, no. 5, pp. 463-470, 2010.

[12] R. J. Fontana, L. B. Seeff, R. J. Andrade et al., "Standardization of nomenclature and causality assessment in drug-induced liver injury: summary of a clinical research workshop," Hepatology, vol. 52, no. 2, pp. 730-742, 2010.

[13] N. Hernández, F. Bessone, A. Sánchez et al., "Profile of idiosyncratic drug induced liver injury in Latin America: an analysis of published reports," Annals of Hepatology, vol. 13, no. 2, pp. 231-239, 2014.

[14] M. B. De Valle, V. Av Klinteberg, N. Alem, R. Olsson, and E. Björnsson, "Drug-induced liver injury in a Swedish University hospital out-patient hepatology clinic," Alimentary Pharmacology and Therapeutics, vol. 24, no. 8, pp. 1187-1195, 2006.

[15] J. M. Leitner, W. Graninger, and F. Thalhammer, "Hepatotoxicity of antibacterials: pathomechanisms and clinical," Infection, vol. 38, no. 1, pp. 3-11, 2010.

[16] M. Smieja, "Current indications for the use of clindamycin: a critical review," Canadian Journal of Infectious Diseases, vol. 9, no. 1, pp. 22-28, 1998.

[17] C. Y. Chang and T. D. Schiano, "Review article: drug hepatotoxicity," Alimentary Pharmacology and Therapeutics, vol. 25, no. 10, pp. 1135-1151, 2007.

[18] T. Ajayi and J. Okudo, "Cardiac arrest and gastrointestinal bleeding: a case of medical heuristics," Case Reports in Medicine, vol. 2016, Article ID 9621390, 4 pages, 2016. 


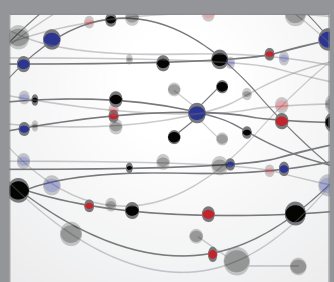

The Scientific World Journal
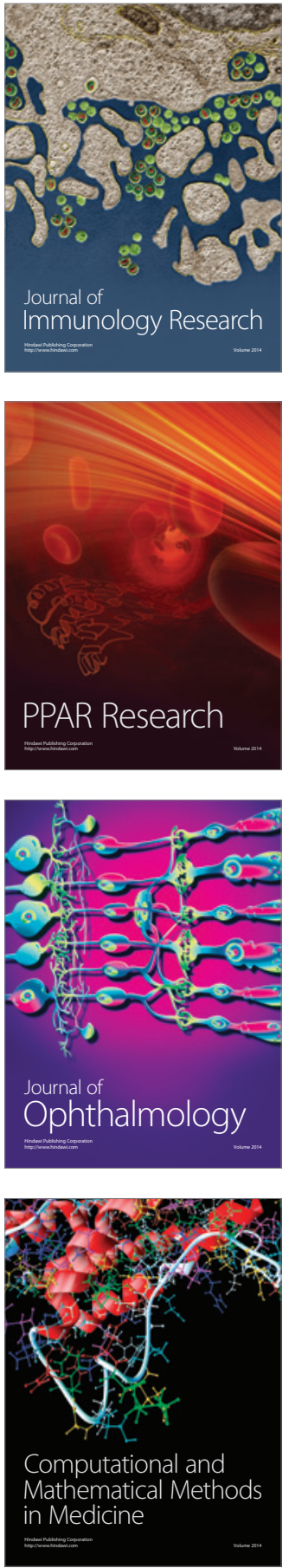

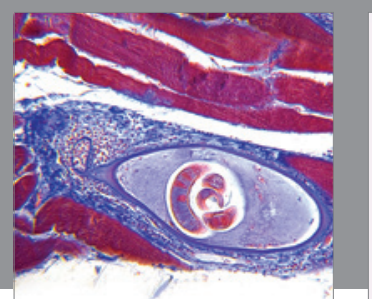

Gastroenterology Research and Practice

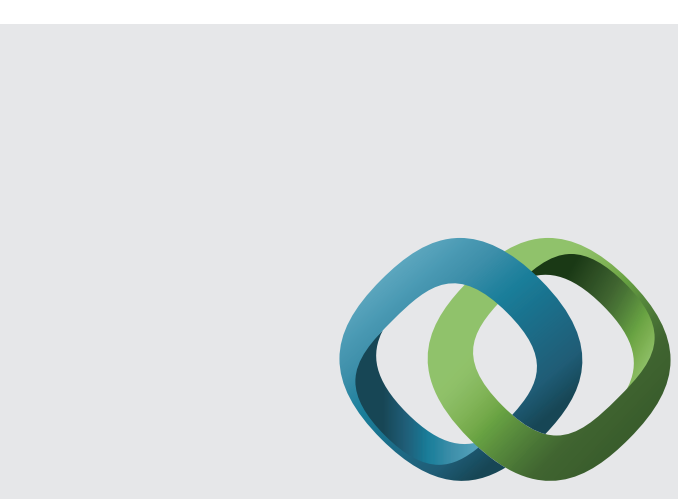

\section{Hindawi}

Submit your manuscripts at

http://www.hindawi.com
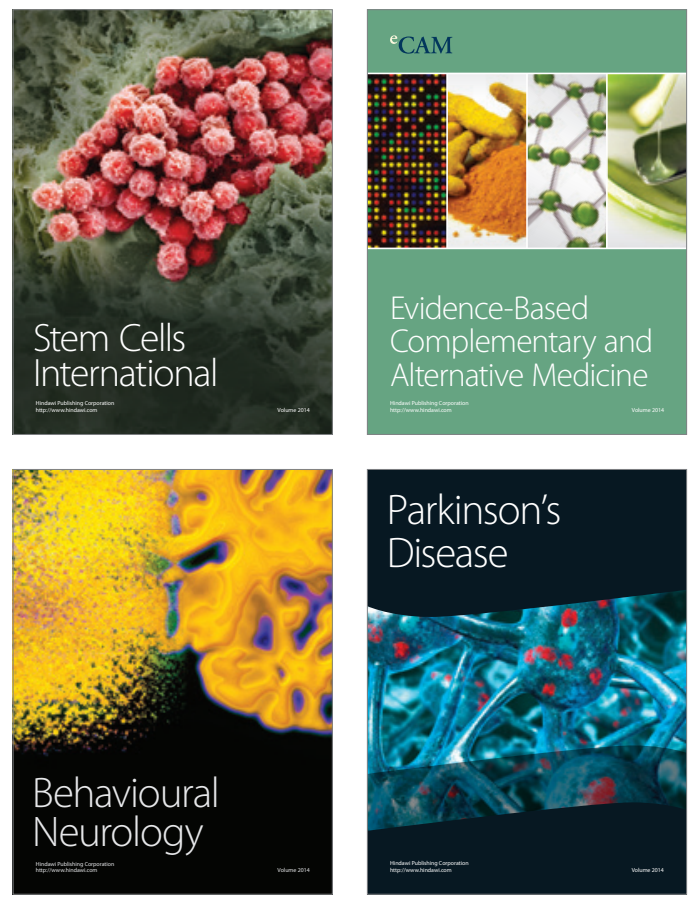
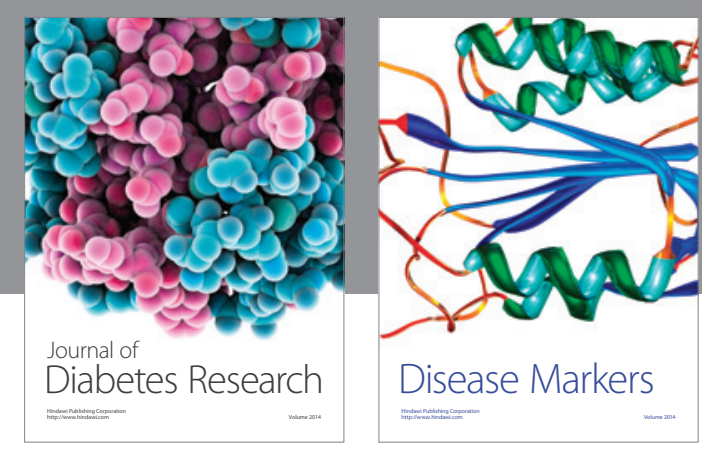

Disease Markers
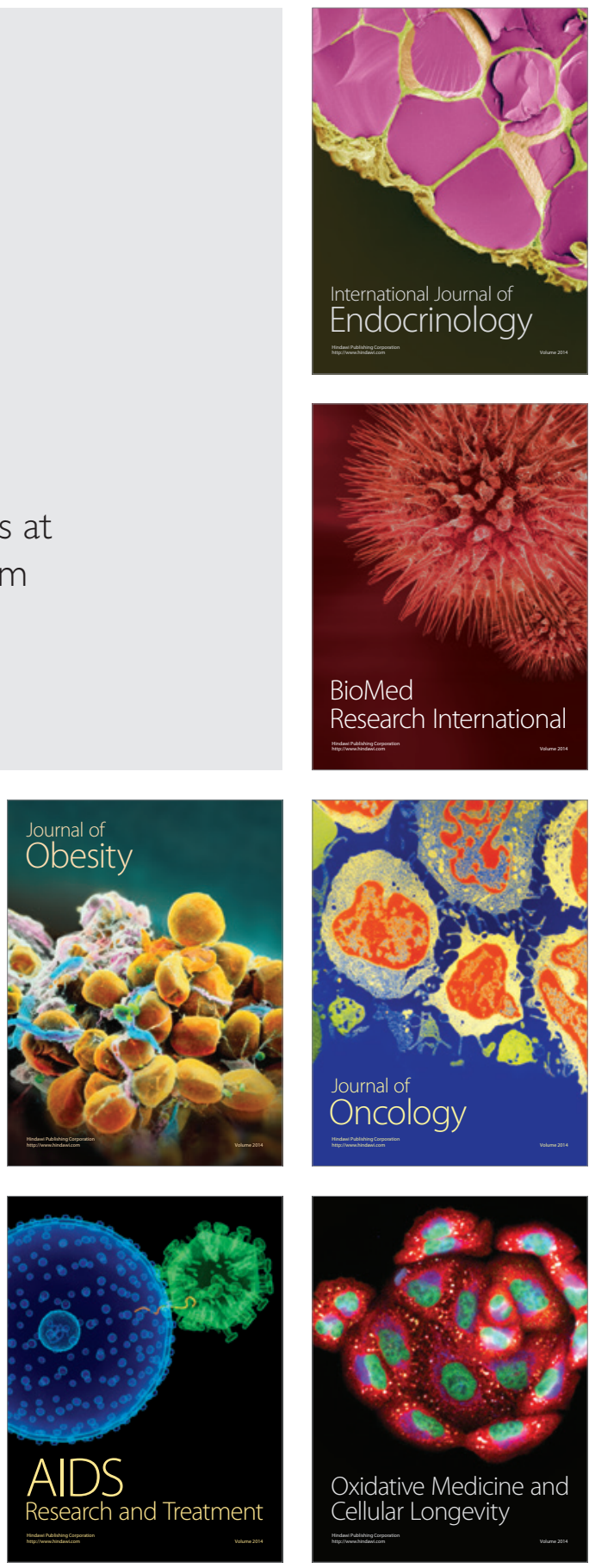